\title{
ADVOCACYEM REDE: EM BUSCA DE MAIOR IMPACTO DO INVESTIMENTO SOCIAL PRIVADO NO BRASIL
}

\author{
Lívia Menezes Pagotto
}

\section{// resumo}

Este artigo tem o objetivo de contextualizar e debater as oportunidades de advocacy em rede no âmbito do investimento social privado no Brasil. Nos últimos anos, práticas de advocacy passaram a ser abordadas de forma mais recorrente pelos principais estudos sobre esse campo e foram incluídas no debate público na busca pela potencialização das ações dos investidores sociais. Temas como o dilema entre financiamento ou operação do advocacy, os processos de monitoramento e avaliação do advocacy, o advocacy no ciclo de políticas públicas e o advocacy em rede tem ganhado relevância. Para o último, foi realizada uma revisão de literatura sobre o tema, uma análise histórica de sua evolução nas edições do Censo GIFE e identificadas iniciativas coletivas de advocacy no âmbito da sociedade civil. Atividades como as do Observatório do Clima, da Coalizão Brasil Clima, Florestas e Agricultura e da Rede de Advocacy Colaborativo evidenciam diversos benefícios: compartilhamento e otimização de recursos, construção de sinergia entre agendas de incidência política, aprendizagem coletiva baseada no levantamento de controvérsias e construção de consenso, potencialização da geração de conhecimento e uso das tecnologias para compartilhamento de informações e execução de estratégias coletivas de advocacy.

| palavras-chave: políticas públicas | sociedade civil | investimento social privado | advocacy. 


\section{artigos \\ GIFE}

\section{OS ARTIGOS GIFE}

A série Artigos GIFE publica reflexões e análises de pesquisadores brasileiros de diferentes áreas do saber com o objetivo de estimular, ampliar e disseminar a produção de conhecimento qualificado sobre o campo da filantropia, do investimento social privado e da sociedade civil no Brasil.

A partir da premissa de que a produção de conhecimento sobre esses temas tem um papel fundamental na expansão, diversificação e qualificação da ação pública pela sociedade, a iniciativa também pretende contribuir para ampliar as conexões entre o setor, as universidades e os centros de pesquisa, promovendo e apoiando o envolvimento de novos atores com a temática e a circulação ampliada de ideias em torno dela.

Este texto integra a segunda edição dos Artigos GIFE, que propõe a publicação mensal de artigos selecionados por meio de chamada pública e convite a autores especialistas em temáticas específicas e relevantes para o setor, buscando apoiar e fomentar a produção de conhecimento sobre o investimento social no Brasil.

O Grupo de Institutos Fundações e Empresas (GIFE) é uma organização sem fins lucrativos que reúne associados de origem empresarial, familiar ou independente que investem em projetos de interesse público. Criado em 1989 como grupo de troca e colaboração e institucionalizado em 1995 por 25 organizações, tornou-se referência no campo da filantropia e do investimento social privado no Brasil.

Nesses mais de 20 anos de atuação, tem contribuído para o fortalecimento de práticas e ações a serviço do bem comum no país, trabalhando para expandir, qualificar e fortalecer o investimento social privado, diversificar e ampliar atores e recursos, criando referências e estimulando boas práticas de gestão, bem como articulando o setor com a sociedade e a agenda pública. Atualmente, o GIFE reúne mais de 150 associados que, somados, aportam mais de $\mathrm{R} \$ 3$ bilhões por ano em projetos próprios e no apoio a projetos de terceiros. 


\section{INTRODUÇÃO}

A dimensão política da atuação de organizações não-governamentais (ONG) é um tema amplamente estudado, principalmente pela ciência política, pela sociologia e pelas relações internacionais, por meio da análise de movimentos sociais e da influência dessas organizações nas políticas públicas e empresariais (PRAKASH; GUGERTY, 2010). Tal dimensão é frequentemente coberta por meio de organizações de advocacy, idealmente motivadas por crenças baseadas em princípios, fincadas em valores compartilhados e orientadas por problemas que exigem ação coletiva. Entre as temáticas sujeitas à atuação em advocacy estão os direitos humanos, a proteção ambiental, os direitos trabalhistas, a religião, a democracia, entre outros (PRAKASH; GUGERTY, 2010).

Advocacy é um conceito amplo que pode dar margem a diferentes interpretações e cujas origens remontam aos movimentos pelos direitos civis nas décadas de 1960 e 1970 nos Estados Unidos (BRELÀZ; ALVES, 2011). Pode ser definido como um conjunto de esforços sistemáticos para promover metas políticas específicas e por seu caráter coletivo, ou seja, um grupo de atores que persegue a ação coletiva (PRAKASH; GUGERTY, 2010). Indivíduos colaboram porque sozinhos enfrentam dificuldades maiores para influenciar as políticas públicas e creem que a ação coletiva por meio de organizações de advocacy é mais efetiva. Essa relação nem sempre é, entretanto, de colaboração: as organizações de advocacy operam em arenas de competição por financiamento, visibilidade na mídia e apoio do eleitorado (PRAKASH; GUGERTY, 2010).

Nos últimos anos, práticas de advocacy no contexto do investimento social privado (ISP) no Brasil passaram a ser abordadas de forma mais recorrente pelos principais estudos sobre esse campo e foram incluídas no debate público na busca pela potencialização das ações dos investidores sociais. Da mesma forma que indivíduos juntam seus recursos e coordenam suas estratégias de influência e incidência política (e competem) para atingir objetivos de forma mais eficiente, organizações da sociedade civil também se reúnem e somam esforços, formando redes de advocacy transnacionais, regionais ou nacionais (KECK; SIKKINK, 1999). Mas, diferentemente da vasta literatura já elaborada sobre indivíduos reunidos para a ação coletiva, há uma demanda pela ampliação do entendimento sobre a atuação em rede de organizações que visam influenciar as políticas públicas. Além disso, o próprio conceito de advocacy tem sido pouco discutido no Brasil, apesar dos numerosos estudos sobre movimentos sociais e organizações da sociedade civil (BRELÀZ; ALVES, 2011). Este artigo pretende contribuir para este debate.

Por meio de uma revisão de literatura, de uma análise histórica da evolução do tema nas edições do Censo do Grupo de Institutos Fundações e Empresas (GIFE) e da identificação de exemplos atuantes no Brasil, buscou-se contextualizar e debater as oportunidades de advocacy em rede no âmbito do ISP. O texto está organizado da seguinte forma: primeiro, há a contextualização do advocacy no campo do ISP no Brasil e a evolução de sua aparição nas edições do Censo GIFE; em segundo lugar, são apresentados exemplos bem-sucedidos no Brasil; em seguida, é feita uma discussão sobre dois aspectos relacionados à emergência de iniciativas de advocacy em rede - (i) os motivos pelos quais elas vêm se tornando um tema e uma prática em ascensão no campo do ISP no Brasil e (ii) os benefícios da ação colaborativa nesse contexto. Por fim, são apresentadas considerações finais.

$1 \mathrm{O}$ precursor da teoria sobre os aspectos mobilizadores de indivíduos para a ação coletiva e para a cooperação em busca de objetivos comuns foi Mancur Olson, economista norte-americano que lançou, em 1971, o livro A Lógica da Ação Coletiva (OLSON, 1971). 


\section{O ADVOCACY NO CONTEXTO DO INVESTIMENTO SOCIAL PRIVADO NO BRASIL}

No contexto do ISP, advocacy pode ser definido como "esforço organizado para mudar as políticas, as práticas e/ou as atitudes por meio de evidências e argumentos de como e por quê a mudança deve acontecer" (AICHER et al., 2010, p. 1) ou como "categoria de atividades - geralmente realizadas por donatários, mas às vezes empreendidas diretamente por fundações - cujo objetivo principal é influenciar a opinião ou as ações das pessoas sobre questões de política ou preocupação pública” (PROSCIO, 2011, p. 4).

Advocacy é uma das formas utilizadas pelo ISP na interação com o poder público, ao lado de iniciativas de alinhamento do investimento às políticas públicas, de avaliação de políticas públicas e de controle social, por exemplo ${ }^{2}$. No caso do advocacy, a decisão de influenciar governos em direção a um objetivo de incidência política definido pelo investidor social pode se traduzir em diversas estratégias, como mostra a Figura 1.

\section{Figura 1 - 0 framework das estratégias de advocacy}

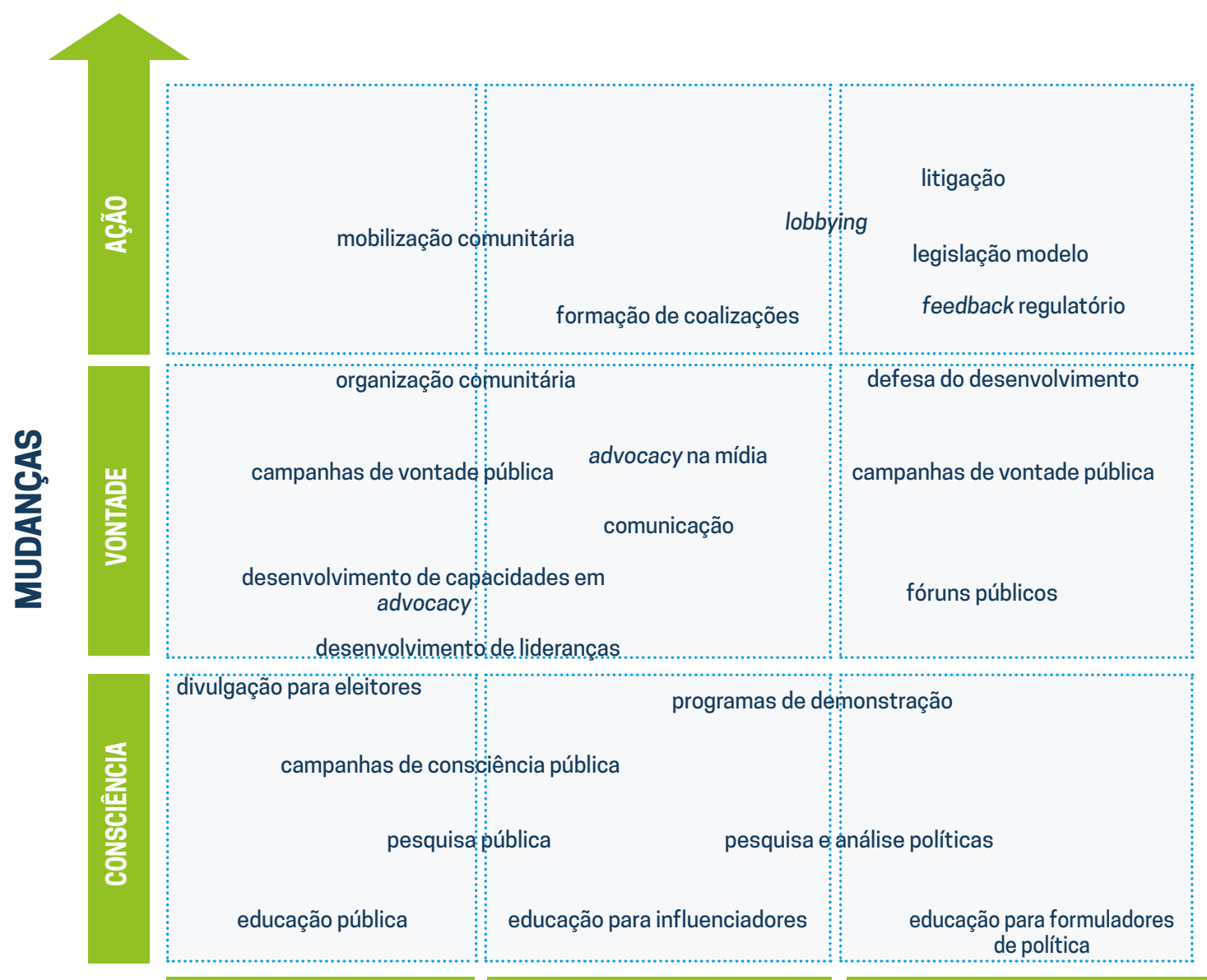

PÚBLICO

INFLUENCIADORES

TOMADORES DE DECISÃO

AUDIÊNCIAS

Fonte: COFFMAN; BEER, 2015. Tradução livre.

2 Somente 14\% dos investidores sociais privados respondentes do Censo GIFE 2016 não consideraram as políticas públicas em sua atuação (GIFE, 2016). 
O desenho da(s) estratégia(s) de advocacy deve considerar duas dimensões. A primeira é a audiência a ser atingida (eixo horizontal da Figura 1), que representa os atores a serem influenciados ou persuadidos no processo político (COFFMAN; BEER, 2015). Eles podem ser o público em geral, influenciadores políticos (mídia e líderes comunitários ou empresariais) ou tomadores de decisão (gestores públicos, juízes e políticos eleitos). A segunda dimensão refere-se às mudanças desejadas (eixo vertical da Figura 1), considerando um espectro de mobilização da consciência, da vontade e da ação no contexto de assuntos de interesse público.

No panorama brasileiro dos últimos anos, o incremento do uso do termo advocacy no campo do ISP, nos principais fóruns de debate e nos estudos sobre as estratégias de atuação, pode ser constatado pela evolução histórica do termo nas edições do Censo GIFE.

A Tabela 1 mostra as ocorrências do termo advocacy no Censo GIFE (GIFE, 2002, 2004, 2006, 2008, 2010, 2013, 2015, 2017). Não há, portanto, uma preocupação de análise qualitativa da atuação das organizações associadas ao GIFE, mas de observação do comportamento do tema nas diferentes edições do Censo. A aparição do termo advocacy no rol de questões feitas por meio do questionário do Censo GIFE enviado aos associados é diferenciada da inclusão das respostas na apresentação dos resultados, em duas colunas.

\section{Tabela 1 - Ocorrências do termo advocacy no questionário e na publicação do Censo GIFE, por edição}

\begin{tabular}{|c|c|c|}
\hline Edição do Censo GIFE & $\begin{array}{c}\text { Número de ocorrências do termo advocacy } \\
\text { no questionário }\end{array}$ & $\begin{array}{l}\text { Número de ocorrências do termo } \\
\text { advocacy na publicação }\end{array}$ \\
\hline $2001-2002$ & - & - \\
\hline 2003-2004 & - & - \\
\hline 2005-2006 & - & - \\
\hline $2007-2008$ & 1 & 2 \\
\hline $2009-2010$ & 4 & 4 \\
\hline 2011-2012 & 4 & 2 \\
\hline 2014 & 4 & 7 \\
\hline 2016 & 8 & 29 \\
\hline
\end{tabular}

Nota-se que, nas três primeiras edições do Censo GIFE, referentes a 2001-2002, 2003-2004 e 20052006, não houve nenhuma ocorrência do termo advocacy. A sua menção inaugural ocorreu na edição 2007-2008, em uma pergunta sobre como as escolhas dos projetos e programas relacionados a jovens era feita pelas organizações, sendo uma das alternativas a atuação em prol de políticas públicas ( $a d-$ vocacy). A partir daí, é possível observar a crescente ocorrência de advocacy nas edições do Censo, culminando em 29 delas em 2016, considerando a atuação dos investidores sociais.

O segundo ponto importante sobre a abordagem do tema advocacy no Censo GIFE é a inclusão do termo ora como área de atuação relacionada a alguma questão específica, ora como estratégia de atuação das organizações associadas ao GIFE. Por exemplo, nas edições 2009-2010 e 2011-2012, o termo advocacy sempre aparece associado ao foco das organizações (formação de jovens, defesa de direitos, cultura, meio ambiente, por exemplo). E, a partir da edição de 2014, passou a aparecer também como ação utilizada nos principais programas para atuação pela causa das organizações e igualmente em outras perguntas referentes à relação das organizações com o poder público e aos objetivos de avaliações e das ações de comunicação. 


\section{EXERCITANDO 0 ADVOCACY EM REDE: EXEMPLOS RECENTES NO BRASIL}

Nos últimos anos, a sociedade civil brasileira se organizou em torno de temas socioambientais no formato de redes e coalizões, cuja busca da atividade em advocacy é centrada nos influenciadores, como audiência, e na ação, como mudança pretendida (Figura 1).

Neste estudo, foi possível identificar cinco redes atuantes no Brasil: o Observatório do Clima (OC), a Coalizão Brasil Clima, Florestas e Agricultura, a Rede de Advocacy Colaborativo (RAC), o Pacto pela Democracia e a Rede de Justiça Criminal. Elas foram selecionadas a partir dos seguintes critérios: atividades em curso em 2019, formação de coalizões e redes entre diferentes organizações, existência de instâncias de coordenação colegiadas, estruturas compartilhadas de secretariado executivo, uso comum de recursos e infraestrutura e formulação conjunta de propostas para intervenção na esfera de políticas públicas.

O OC foi fundado em 2002 em forma de coalizão de organizações da sociedade civil brasileira para discutir mudanças climáticas. Desde então, contribui para o debate público por meio de capacitação para a ação, elaboração de propostas e geração de dados públicos sobre assuntos relacionados a mudanças climáticas no Brasil (OBSERVATÓRIO DO CLIMA, 2019).

A Coalizão Brasil Clima, Florestas e Agricultura tem como missão articular e facilitar ações para que o país promova um novo modelo de desenvolvimento econômico pautado na economia de baixo carbono e, dessa maneira, responda aos desafios das mudanças climáticas. Iniciada em 2015 como movimento multisetorial, hoje conta com mais de 150 organizações que se reúnem periodicamente para adotar posicionamentos comuns a elas e propor ações (COALIZÃO BRASIL CLIMA, FLORESTAS E AGRICULTURA, 2019).

A RAC surgiu formalmente em 2018 com o intuito de reunir organizações da sociedade para a realização de advocacy focado em quatro temas debatidos em grupos de trabalho: questões socioambientais, direitos humanos, transparência e integridade e nova economia. Por meio de ações de fortalecimento e colaboração entre organizações da sociedade civil, a rede busca conectar interesses coletivos e difusos da sociedade civil e estreitar a sua distância em relação ao parlamento brasileiro. Três aspectos motivaram a criação da RAC: a demanda por mais acesso ao conhecimento sobre estratégias efetivas de advocacy e lobby ${ }^{3}$, inclusive estratégias utilizadas por movimentos opostos aos princípios da RAC; a deficiência das capacidades das organizações em relação ao uso de inteligência de dados relacionados aos processos legislativos; e a compreensão de como a comunicação pode ser um meio efetivo para aproximar o cotidiano dos cidadãos com o que acontece no Congresso (REDE DE ADVOCACY COLABORATIVO, 2019).

O Pacto pela Democracia surgiu em 2018 como uma iniciativa da sociedade civil brasileira plural, apartidária, aberta a todos os cidadãos e voltada à defesa e ao aprimoramento da vida política e democrática no Brasil. Formado por um grupo de organizações, movimentos e atores relevantes na sociedade, o Pacto busca afirmar um espaço comum para a expressão do compromisso de resgatar e aprofundar práticas e valores democráticos diante dos inúmeros desafios enfrentados ao longo dos últimos anos no país. As ações são orientadas em torno de três eixos norteadores: promoção da cultura democrática, com a valorização do pluralismo, da tolerância e do engajamento cidadão; defesa e revigoramento da institucionalidade democrática e os princípios, liberdades e direitos fundamentais expressos na Constituição Federal; e fortalecimento da articulação de redes e de ação conjunta na sociedade civil e no universo político em defesa da construção democrática (PACTO PELA DEMOCRACIA, 2019).

A Rede Justiça Criminal, criada em 2010, é um grupo composto por oito ONG brasileiras e tem como objetivo contribuir e influenciar o debate em temas pertinentes ao sistema de justiça criminal. A articulação permanente entre as organizações-membro permite a troca de experiências, a colaboração entre organizações com diferentes especialidades e o impulso mútuo em direção a objetivos compartilhados. A Rede Justiça Criminal desenvolve uma agenda baseada em eixos de atuação e temas prioritários (dados e transparência, direitos e garantias, violência institucional), monitora projetos

3 Lobby é uma das estratégias possíveis de advocacy. Ele é “o ato de influenciar tomadores de decisão política, seja ela do executivo, legislativo ou judiciário, para que a decisão atenda um determinado interesse” (CASTRO, 2016, p. 38). 
de lei e políticas na área de justiça criminal e desempenha o trabalho de advocacy junto aos Poderes Legislativo e Executivo e à sociedade civil (REDE JUSTIÇA CRIMINAL, 2019).

Além dessas cinco redes e, especificamente, no campo do ISP, o GIFE compõe uma rede expressiva com atuação em advocacy focada no fortalecimento das organizações da sociedade civil desde 2010. Junto com um grupo de organizações, redes, coletivos e movimentos sociais, o GIFE integra uma Plataforma por um Novo Marco Regulatório para as Organizações da Sociedade Civil (MROSC), que passou a articular diferentes atores para o desenvolvimento e implementação de um marco legal que proporcionasse um ambiente jurídico mais seguro e favorável à sua atuação.

Nas cinco iniciativas de advocacy em rede no Brasil puderam ser observadas algumas características comuns. Todas apresentam um conjunto de princípios e valores compartilhados, o que representa o seu caráter normativo (PRAKASH; GUGERTY, 2010). Fortalecer a democracia, articular um novo modelo de desenvolvimento econômico e estreitar a relação entre os cidadãos e o parlamento são exemplos disso. Da mesma forma, as redes analisadas estão orientadas por problemas que demandam ação coletiva, tais como as mudanças climáticas, a economia de baixo carbono, a promoção da cultura democrática e a contribuição ao sistema de justiça criminal brasileiro.

A seguir, são discutidos dois aspectos relacionados à emergência de iniciativas de advocacy em rede no Brasil: (i) os motivos pelos quais elas vêm se tornando um tema e uma prática em ascensão no campo do ISP no Brasil, e (ii) os benefícios da ação colaborativa nesse contexto.

\section{POR QUE ESTAMOS FALANDO MAIS SOBRE ADVOCACY NO CAMPO DO INVESTIMENTO SOCIAL PRIVADO NO BRASIL?}

Três motivos foram identificados como motivadores da popularidade do tema advocacy no Brasil: o cenário político-institucional no país, o impacto do ISP em relação aos recursos investidos e o aspecto paradoxal relacionado ao propósito do ISP e do advocacy.

Em primeiro lugar, há o cenário político-institucional brasileiro nos últimos anos. Não é recente o debate público sobre a urgência da reforma do sistema político e da revisão do papel dos partidos políticos em relação às agendas nacionais prioritárias. Passou a haver, inclusive, o questionamento acerca da perda de capacidade de convergência e da legitimidade das agendas defendidas pelos partidos e do processo de suas constituições a partir de demandas sociais negociadas de forma qualificada e representativa na esfera pública. Nesse vácuo de legitimidade e representatividade dos partidos políticos, a sociedade civil e os investidores sociais privados se depararam com a oportunidade de contribuir para a extração e depuração de agendas sociais prioritárias, de forma menos institucionalizada e com maior diluição da concentração de poder.

Nesse mesmo contexto, os mais recentes abalos sofridos pelas bases fundantes da democracia - tais como a participação social e o exercício pleno da defesa de direitos - provocaram uma série de autoanálises por parte dos investidores sociais privados brasileiros a respeito de sua real contribuição em relação aos marcos democráticos e ao papel das políticas públicas. Residiria aqui uma segunda razão para a proeminência do advocacy: uma reflexão do próprio impacto do ISP em relação aos recursos investidos. As práticas de advocacy - originalmente características dos movimentos ambientalistas com caráter combativo contra a pobreza nos países em desenvolvimento, contra o desmatamento nas florestas tropicais, contra o imobilismo político e cidadão em relação às mudanças climáticas etc. -, passaram a ser exercidas no campo do ISP com o intuito de garantir a permanência das conquistas já alcançadas em termos de progresso social, conservação ambiental e desenvolvimento econômico e a confirmação de sua própria agenda. 
Por fim, o terceiro motivo para a popularidade das iniciativas de advocacy no ISP é reiterativo. Assumindo que o ISP é "o repasse voluntário de recursos privados de forma planejada, monitorada e sistemática para projetos sociais, ambientais, culturais e científicos de interesse público" (GIFE, 2019), ele seria alinhado à natureza das ações de advocacy, já que ambos convergem em termos de finalidade: influenciar e contribuir para ações de interesse público, com impacto e transformação social. Isso significa dizer que as ações de incidência política sejam uma das formas mais extremas de realização do investimento feito por empresas, institutos e fundações voltados ao interesse público.

\section{O ADVOCACY EM REDE: OS BENEFICIOS DE SE FAZER JUNTOS}

As redes e as coalizões de incidência política ganham maior relevância em momentos de fragilização do tecido político-institucional e de atuação desfavorável das organizações da sociedade civil. Mesmo em contextos mais favoráveis, são diversas as motivações para o advocacy em rede.

Há claramente uma oportunidade de compartilhamento e otimização de recursos humanos e financeiros. As atividades de monitoramento dos processos legislativos e de assessoria jurídica para a constituição de novos marcos legais são exemplos de processos custosos, mas que podem ser compartilhados por organizações que trabalham em temas convergentes. Profissionais especialistas nas atividades de advocacy podem ter a sua atuação potencializada com maior aporte de recursos, além de perceberem maior contribuição para um grupo mais amplo de organizações.

Outra razão é a sinergia entre agendas para a incidência política, podendo contribuir para seu fortalecimento e ganho de legitimidade (DIOKNO-PASCUAL, 2002) perante a sociedade e, principalmente, os agentes públicos. Dessa sinergia pode derivar um processo de aprendizagem coletivo, baseado na identificação de controvérsias e na construção de consensos. Isso pode levar à lapidação das estratégias de atores da sociedade civil para influenciar a sua capacidade de propor ou reforçar marcos regulatórios e processos sociais de maneira mais efetiva.

O trabalho em rede pode também potencializar a influência política ao longo de todo o ciclo de políticas públicas - definição da agenda, implementação e avaliação das políticas públicas (COFFMAN, 2008) uma vez que seus diversos participantes podem encadear ações relacionadas a uma única causa a partir de suas expertises. Por exemplo, o complemento da capacidade de uma organização de formular uma política pública por outra mais dedicada à sua implementação gera continuidade das ações de advocacy.

Para lidar com contextos complexos, como são aqueles típicos na atuação dos investidores sociais privados, demanda-se conhecimentos diversos, formais e informais. A redução da pobreza e da desigualdade social, a conservação ambiental, a constituição de espaços urbanos mais saudáveis e sustentáveis são, por natureza, temas interdisciplinares. A inteligência coletiva das redes reunidas em torno de causas comuns tem o poder de explorar melhor as questões em jogo, de propiciar estratégias mais efetivas de incidência nas políticas públicas, além de envolver possivelmente tomadores de decisão na formulação das propostas.

influência política ao longo

de todo o ciclo de políticas

públicas - definição da

agenda, implementação

e avaliação das políticas públicas
Quando agindo em rede paira, ainda, uma questão relacionada ao ISP e ao advocacy no Brasil: o dilema entre o financiamento de organizações de advocacy ou a operação direta do advocacy por um instituto ou fundação. Sobre essa questão, uma publicação do GrantCraft sugere alguns fatores que podem contribuir para a tomada de decisão: (i) quem tem um melhor conhecimento sobre as questões substantivas, do processo de políticas públicas e dos meios de in-

fluenciar as decisões públicas; (ii) se as restrições legais sobre uma atividade são diferentes de outra, e se essas diferenças afetam o tipo de advocacy que pode ser realizado; (iii) quem é mais capaz de dedicar recursos humanos, tempo e resistência para assumir um papel público sobre o assunto; (iv) quem daria maior peso ou autoridade - técnica, política ou moral - às opiniões expressas; e (v) quem 
está disposto e é capaz de aceitar a publicidade - e talvez a controvérsia - que pode resultar de um papel publicamente visível (PROSCIO, 2011).

Uma última razão está conectada ao uso de tecnologias para o compartilhamento de informações e para a construção conjunta de estratégias de advocacy como outro ganho da atuação coletiva para influenciar as políticas públicas. Um exemplo disso é o aplicativo 342Amazônia, lançado em 2019 pelo Greenpeace e pelo Mídia Ninja como a primeira ferramenta ativista ambiental do Brasil voltada à ação e à mobilização para a proteção da Amazônia, a natureza e as pessoas. Da mesma forma, os recursos tecnológicos são fundamentais para a comunicação e a influência da opinião pública que, conforme a Figura 1, são estratégias do quadrante inferior esquerdo, para aumentar a conscientização do público em geral.

\section{CONSIDERAÇÕES FINAIS}

O contexto político e institucional atual brasileiro demanda da sociedade civil estratégias ainda mais inovadoras para afirmar e ampliar o repertório de políticas públicas pautadas pela equidade social e pelo equilíbrio entre o desenvolvimento econômico e a conservação ambiental. Ações de advocacy ganharam ainda mais relevância principalmente para a manutenção de conquistas históricas no campo social frente às narrativas populistas existentes no Brasil nos dias de hoje. Contra a série de retrocessos, o esforço coletivo pautado em redes e movimentos recentes de advocacy é um exercício para a construção coletiva de agendas e para a potencialização da capacidade de incidência política.

A atuação do ISP no Brasil na interação com as políticas públicas espraia-se pelas esferas federal, estadual e municipal de governo. Isso representa uma capilaridade potente para a proposição de transformações nos marcos reguladores e legisladores da vida em coletividade. Investidores sociais agindo em territórios comuns Brasil afora em temáticas convergentes são atores fundamentais para a identificação de oportunidades nos diferentes níveis do governo.

Por outro lado, o advocacy ligado ao ISP, assim como as próprias causas e ações desenvolvidas pelos investidores sociais, também enfrenta desafios ligados à legitimidade das agendas e das ações propostas, já que se trata de uma forma de representação não-eleitoral. Daí a importância da atuação em rede como aspecto favorável ao incremento da legitimidade para a influência política, mitigando eventuais conflitos de interesse e construindo consensos mais coletivos. Igualmente relevantes são os esforços de monitoramento e avaliação dos impactos obtidos por meio da incidência política. Para tanto, ainda há no Brasil uma lacuna em termos de desenvolvimento das capacidades necessárias para a sua realização. O monitoramento e a avaliação dos impactos das atividades de advocacy exigem a ponderação entre métricas qualitativas e quantitativas, conjugando a apuração dos objetivos, estratégias e resultados de curto, médio e longo prazos. Na atuação em rede, o desafio maior desse exercício é a identificação da contribuição de cada organização pertencente à rede e também de cada dimensão do impacto causado. Surgem daí as oportunidades para iniciativas coletivas e compartilhadas de avaliação de impacto para a apuração mais precisa da contribuição do ISP para a sociedade.

Por fim, é necessário reconhecer uma limitação clara deste artigo derivada da abordagem mais otimista adotada acerca da atuação das organizações de ISP em rede. Nem sempre o advocacy e o ISP advogam em favor de agendas de interesse público de cunho progressista, e frequentemente há a constatação de divergências e conflitos entre ambos. Há, portanto, uma agenda de pesquisa a ser explorada a partir de experiências em curso no Brasil uma vez que, conforme mencionado na introdução deste artigo, as organizações e as redes de advocacy colaboram, mas também competem, por visibilidade e por recursos. 


\section{REFERÊNCIAS}

AICHER, Rachel; NAPIER, Fiona; PICKARD, Russell. An introductory guide to successful advocacy. Open Society Foundations, 2010. Disponível em: https://www.opensocietyfoundations.org/publications/evidence-messages-change-introductory-guide-successful-advocacy. Acesso em: 06/07/2019.

BRELÀZ, Gabriela de; ALVES, Mário Aquino. Deliberative democracy and advocacy: lessons from a comparative perspective. Canadian Journal of Administrative Sciences. Revue Canadienne des Sciences de l'Administration, n. 28, p. 202-216, 2011. DOI: 10.1002/CJAS.206.

CASTRO, Daniela. Advocacy. Como a sociedade pode influenciar os rumos do Brasil. 2016. São Paulo: SG-Amarante Editorial.

COALIZÃO BRASIL CLIMA, FLORESTAS E AGRICULTURA. Quem somos. 2019. Disponível em: http://www.coalizaobr.com.br/home/ index.php/sobre-a-coalizao/quem-somos. Acesso em: 16/07/2019.

COFFMAN, Julia. Foundations and public policy grantmaking. The James Irving Foundation. 2008. Disponível em: https://community-wealth. org/sites/clone.community-wealth.org/files/downloads/paper-coffman.pdf. Acesso em: 05/07/2019.

COFFMAN, Julia; BEER, Tanya. The advocacy strategy framework. A tool for articulating an advocacy theory of change. Center for Evaluation Innovation, 2015. Disponível em: http://www.pointk.org/resources/files/Adocacy_Strategy_Framework.pdf. Acesso em: 05/07/2019

DIOKNO-PASCUAL, Maria Teresa. Development and advocacy. In: EADE, Deborah (ed.). Development and advocacy. Oxford: Oxfam GB, 2002, p. 1-6. Disponível em: https://oxfamilibrary.openrepository.com/bitstream/handle/10546/121187/bk-development-advocacy-010202en.pdf? sequence=5\&isAllowed=y. Acesso em: 05/07/2019.

GIFE - Grupo de Institutos Fundações e Empresas. Censo GIFE 2001-2002. 2002. Documento em pdf.

GIFE - Grupo de Institutos Fundações e Empresas. Censo GIFE 2003-2004. 2004. Documento em pdf.

GIFE - Grupo de Institutos Fundações e Empresas. Censo GIFE 2005-2006. 2006. Disponível em: https://sinapse.gife.org.br/download/ censo-gife-2005-2006. Acesso em: 02/07/2019.

GIFE - Grupo de Institutos Fundações e Empresas. Censo GIFE 2007-2008. 2008. Disponível em: https://sinapse.gife.org.br/download/ censo-gife-2007-2008. Acesso em: 02/07/2019.

GIFE - Grupo de Institutos Fundações e Empresas. Censo GIFE 2009-2010. 2010. Disponível em: https://sinapse.gife.org.br/download/ censo-gife-2009-2010. Acesso em: 02/07/2019.

GIFE - Grupo de Institutos Fundações e Empresas. Censo GIFE 2011-2012. 2013. Disponível em: https://sinapse.gife.org.br/download/ censo-gife-2011-2012. Acesso em: 02/07/2019.

GIFE - Grupo de Institutos Fundações e Empresas. Censo GIFE 2014. 2015. Disponível em: https://sinapse.gife.org.br/download/censogife-2014. Acesso em: 02/07/2019.

GIFE - Grupo de Institutos Fundações e Empresas. Censo GIFE 2016. 2017. Disponível em: https://sinapse.gife.org.br/download/censogife-2016. Acesso em: 02/07/2019.

GIFE - Grupo de Institutos Fundações e Empresas. Investimento social privado. 2019. Disponível em: https://gife.org.br/investimento-socialprivado/. Acesso em: 02/07/2019.

KECK, Margaret E.; SIKKINK, Kathryn. Transnational advocacy networks in international and regional politics. 1999. International Social Science Journal, v. 51, n. 159, p. 89-101, 1999. https://doi.org/10.1111/1468-2451.00179.

OBSERVATÓRIO DO CLIMA. Nossa história. 2019. Disponível em: http://www.observatoriodoclima.eco.br/nossa-historia/. Acesso em: 16/07/2019.

OLSON, Mancur. A lógica da ação coletiva. Tradução de Fábio Fernandez. São Paulo: EdUSP, 1999.

PACTO PELA DEMOCRACIA. Sobre o Pacto pela Democracia. 2019. Disponível em: https://www.pactopelademocracia.org.br/o-pacto. Acesso em: 16/07/2019.

PRAKASH, Aseem; GUGERTY, Mary Kay. Advocacy organizations and collective action: an introduction. In: PRAKASH, Aseem; GUGERTY, Mary Kay (eds.). Advocacy organizations and collective action. New York: Cambridge University Press, 2010. 318 pp.

PROSCIO, Tony. Advocacy funding: the philanthropy of changing minds. GrantCraft, 2011. Disponível em: http://grantcraft.org/content/ guides/advocacy-funding/. Acesso em: 06/07/2019.

REDE DE ADVOCACY COLABORATIVO. Apresentação institucional. 2019. Acesso em: 16/07/2019.

REDE JUSTIÇA CRIMINAL. O que fazemos. 2019. Disponível em: https://redejusticacriminal.org/pt/what-we-do-2/. Acesso em: 16/07/2019. 


\section{A AUTORA}

\section{Lívia Menezes Pagotto}

Doutoranda em Administração Pública e Governo na FGV-EAESP, Mestre em Governança Ambiental pela Albert-Ludwigs Universität Freiburg e Cientista Social pela PUC-SP. Atualmente é gerente de conhecimento e advocacy do Instituto Arapyaú. 


\title{
artigos GIFE
}

Supervisão: José Marcelo Zacchi e Gustavo Bernardino

Coordenação: Graziela Santiago

Produção editorial: Gleice Regina Guerra

Apoio: Carolina Magosso

Diagramação: Clarissa Sitó

Impressão: Forma Certa

ISSN: 2674-8061

DOI: doi.org/10.33816/gife.20190102a1

(C) 2019 GIFE - Grupo de Institutos Fundações e Empresas

\section{(C) (i) ()}

Este material é disponibilizado sob a licenca Creative Commons Atribuição Não Comercial 4.0 Internacional. http://creativecommons.org/licenses/by-nc/4.0

As opiniões e análises expressas nesta publicação não necessariamente refletem as do GIFE.

\author{
Apoio institucional \\ Fundação Ford \\ Fundação Lemann \\ Instituto C\&A \\ Instituto Unibanco
}

QGIFE 